\title{
Características clínicas y microbiológicas de las infecciones de piel y tejidos blandos por Staphylococcus aureus en niños de un hospital en Medellín durante los años 2013 a 2015
}

\author{
Lina María Castaño-Jaramillo, C. Beltrán-Arroyave, L.C. Santander-Peláez, A. M. Vélez-Escobar, \\ C. G. Garcés-Samudio y Mónica Trujillo-Honeysberg
}

Clinical and microbiological characteristics of skin and soft tissue infections caused by Staphylococcus aureus in children in a hospital in Medellin from 2013 to 2015

Background: Skin and soft tissue infections (SSTI) are very common in children and Staphylococcus aureus is the main agent, with an increase of methicillin resistant strains (MRSA) in recent years. Aim: To identify the frequency of MRSA in skin and soft tissue infections (SSTI) in children from a high complex hospital in Medellin, Colombia. Methods: This is a descriptive, retrospective study, information was obtained from medical records. We included patients younger than 18 years with SSTI due to $S$. aureus who did not meet criteria for invasive disease. Results: The prevalence of MRSA in this population was $31 \%$. The main diagnosis was cutaneous abscess (68\%), followed by surgical site infection (15\%) and non-purulent cellulitis $(6 \%)$. Eighty five percent of the patients had at least 1 comorbidity. All isolates were sensitive to rifampicin and cotrimoxazole and $8 \%$ of the isolates were resistant to clindamycin. There was a higher prevalence of MRSA in patients under 2 years compared to older (60 vs 23\%, p =0,0109). Conclusion: In view of the high prevalence of MRSA in SSTI, empirical treatment with adequate coverage for MRSA is recommended, especially for patients under 2 years of age.

Key words: Staphylococcus aureus, skin and soft tissue infections, methicillin-resistant Staphylococcus aureus, rifampin, cotrimoxazole, clindamycin.

Palabras clave: Staphylococcus aureus, infecciones piel y tejidos blandos, Staphylococcus aureus resistente a meticilina, rifampicina, cotrimoxazol, clindamicina.

\section{Introducción}

as infecciones de piel y tejidos blandos (IPTB) son altamente prevalentes en la población pediátrica, $\checkmark$ con un aumento en su incidencia en los últimos años ${ }^{1}$; especialmente en la forma de abscesos cutáneos. Hasta $70 \%$ de éstas son causadas por Staphylococcus aureus resistente a meticilina adquirido en la comunidad $(\mathrm{SARM}-\mathrm{AC})^{1-5}$.

Se define IPTB como los cambios inflamatorios en la epidermis, dermis, tejido celular subcutáneo, fascia profunda o músculo, producidos por un agente infeccioso ${ }^{6}$. La presentación de estas infecciones se debe a la ruptura del equilibrio entre el hospedero, los microorganismos que colonizan la piel y el ambiente ${ }^{7,8}$. También pueden ser la manifestación de infecciones sistémicas con diseminación hematógena ${ }^{9}$.

$\mathrm{Su}$ presentación clínica es variable y depende del microorganismo causal, del mecanismo invasor en la piel y del compromiso de las estructuras anatómicas subyacentes $^{8}$. Las lesiones pueden ser clasificadas según el sitio anatómico de la infección como superficiales (impétigo, forúnculos, celulitis) o profundas (abscesos, piomiositis, fascitis necrosante). Según su gravedad, se pueden clasificar como complicadas y según la presencia de exudado, como purulentas 9 . También se pueden clasificar como primarias cuando se presentan en piel sana con pequeñas lesiones como picaduras o microtraumas y secundarias cuando se presenta en piel alterada como en quemaduras, heridas, etc. ${ }^{6,7}$.

El diagnóstico de las IPTB es clínico y en la mayoría de los casos no se obtienen aislados microbiológicos. El agente etiológico más común es $S$. aureus; en Estados Unidos de América (E.U.A.) han reportado SARM hasta en $30 \%$ de los $\operatorname{casos}^{10}$. En Colombia, hay series que reportan que hasta $69 \%$ de las infecciones provocadas por S. aureus son causadas por $\mathrm{SARM}^{7}$ y la localización más frecuente de estas infecciones es en la piel. Aproximadamente, 25 a $40 \%$ de la población está colonizada por este microorganismo ${ }^{10}$.

El tratamiento antimicrobiano es frecuentemente empírico y su elección se basa en la presentación clínica y la epidemiología local.

Los estudios sobre prevalencia de estas infecciones en
Universidad CES Medellín-Colombia (LMCJ LCSP, AMVE). Hospital Pablo Tobón Uribe (CGGS, MTH) Universidad de Antioquia (CBA).

Los autores declaran no tener conflicto de intereses.

No se obtuvo financiación alguna. Recibido: 27 de febrero de 2017 Aceptado: 25 de mayo de 2017

Correspondencia a: Lina María Castaño Jaramillo linipediatria@gmail.com 
niños en Colombia son escasos y la mayoría de ellos no reportan los aislamientos microbiológicos.

\section{Objetivo}

Definir la prevalencia de $S$. aureus sensible a meticilina (SASM) y SARM en pacientes con IPTB no invasora causada por $S$. aureus en el Servicio de Pediatría de un hospital de cuarto nivel durante tres años. Como objetivos secundarios se realizó la caracterización clínica de la población en la que se obtuvieron los aislados y el perfil de sensibilidad a otros antimicrobianos.

\section{Materiales y Métodos}

Se trata de un estudio descriptivo, retrospectivo, a partir de revisión de historias clínicas de pacientes con infección por $S$. aureus, identificados a través de la base de datos proporcionada por el Laboratorio de Microbiología del Hospital Pablo Tobón Uribe (HPTU), un hospital de cuarto nivel de complejidad, ubicado en la ciudad de

\begin{tabular}{|c|c|c|c|c|}
\hline & SASM (49) & SARM (22) & Unidad & \\
\hline \multicolumn{5}{|l|}{ Características de la población } \\
\hline Femenino & 49 & 55 & $\%$ & \\
\hline Masculino & 51 & 45 & $\%$ & \\
\hline Edad (media) & 7,5 & 4,4 & Años & $p=0,008$ \\
\hline Sin co-morbilidades & 12,2 & 22,7 & $\%$ & \\
\hline 1 co-morbilidad & 61,2 & 40,9 & $\%$ & $p=0,252$ \\
\hline 2 co-morbilidades & 26,5 & 36,4 & $\%$ & $p=0,721$ \\
\hline Infecciones recurrentes & 10,2 & 22,7 & $\%$ & \\
\hline \multicolumn{5}{|l|}{ Diagnóstico } \\
\hline Celulitis & 4,1 & 9,1 & $\%$ & $p=0,583$ \\
\hline Absceso & 65,3 & 72,7 & $\%$ & $p=0,594$ \\
\hline Infección de sitio quirúrgico & 16,3 & 13,6 & $\%$ & \\
\hline Impétigo & 4,1 & 4,5 & $\%$ & \\
\hline Miositis & 2,0 & 0 & $\%$ & \\
\hline Otro & 8,2 & 0 & $\%$ & \\
\hline \multicolumn{5}{|l|}{ Procedencia del cultivo } \\
\hline Aspirado & 22,5 & 31,8 & $\%$ & \\
\hline Secreción & 44,9 & 40,9 & $\%$ & \\
\hline Biopsia & 32,6 & 27,2 & $\%$ & \\
\hline \multicolumn{5}{|l|}{ Conducta } \\
\hline Toma de hemocultivos & 24,5 & 13,6 & $\%$ & $p=0,363$ \\
\hline Drenaje quirúrgico & 55,1 & 54,2 & $\%$ & $p=1,000$ \\
\hline \multicolumn{5}{|l|}{ Tratamiento dirigido } \\
\hline$\beta$-lactámicos & 80 & 10 & $\%$ & \\
\hline Clindamicina & 11 & 43 & $\%$ & \\
\hline Vancomicina & 7 & 0 & $\%$ & \\
\hline Cotrimoxazol & 4 & 43 & $\%$ & \\
\hline Otros & 4 & 4 & $\%$ & \\
\hline
\end{tabular}

Medellín (Colombia). El protocolo fue aprobado por el Comité de Investigaciones del hospital.

Se analizaron todos los aislamientos de $S$. aureus desde el 1 de enero de 2013 hasta el 31 de diciembre de 2015 . Se incluyeron los pacientes bajo 18 años de edad, con IPTB que no presentaron enfermedad invasora, definida por el aislamiento de $S$. aureus de un sitio corporal estéril ${ }^{11}$, según los criterios de $\mathrm{CDC}^{12}$. Se obtuvieron variables demográficas (edad, género), clínicas (tipo de infección, presencia de co-morbilidades, necesidad de drenaje quirúrgico, antecedente de infecciones recurrentes en piel, antimicrobiano(s) indicado (s) empírico y dirigido), de laboratorio (PCR [proteína C reactiva, positiva $>1 \mathrm{mg} / \mathrm{dL}$ ] y antibiograma) a través de un cuestionario previamente diseñado.

Todas las infecciones donde se obtuvo secreción purulenta de manera espontánea, mediante drenaje o aspiración, se catalogaron como absceso. También se incluyó el resultado de la prueba de disco D que predice la resistencia inducible a clindamicina a partir de eritromicina. Estos datos fueron analizados mediante el programa IBM SPSS Statistics ${ }^{\circledR}$.

\section{Resultados}

Se analizaron 71 episodios de IPTB por $S$. aureus de pacientes, desde un mes hasta 17 años de edad, que cumplían los criterios de inclusión. Las características principales de los pacientes se muestran en la Tabla 1. El aislamiento de SARM tuvo una prevalencia de $31 \%$. La edad promedio fue de 6,5 años, con diferencia significativa entre ambos grupos (7,5 años en grupo SASM vs 4,4 años en el grupo SARM (p: 0,008). Se encontró mayor prevalencia de SARM en lactantes comparado con los mayores de 2 años (60 vs 23\%, p: 0,0109).

Los pacientes tuvieron distribución similar por género. El principal diagnóstico fue absceso cutáneo, en $68 \%$, seguido por infección de sitio operatorio en $15 \%$ y celulitis no purulenta en $6 \%$. Se presentaron 48 abscesos, sin diferencia entre aislamiento de SASM y SARM (p: 0,05944).

La duración media del tratamiento fue 10 días, con una estancia hospitalaria promedio de 22 días, pero cabe recalcar que la mayoría de los pacientes $(85 \%)$ presentaban alguna co-morbilidad en el momento de la IPTB. Las más frecuentemente asociadas fueron trauma (24\%), seguida de neoplasia (21\%), alteraciones inmuno-hematológicas (19\%), alteraciones gastrointestinales $(15,5 \%)$ y alteraciones genéticas o neuro-metabólicas $(15,5 \%)$.

Treinta por ciento de los pacientes con aislamiento de SASM tenía antecedente de trauma vs 9\% en SARM, diferencia no significativa estadísticamente (p: 0,0710).

Se encontró antecedente de infecciones recurrentes en piel en $14 \%$ de los pacientes, sin diferencia significativa 
entre ambos grupos, 10,2\% en el grupo de SASM y $22,7 \%$ en el grupo de SARM (p: 0,2663).

En 59\% de los casos a los que se les realizó PCR, ésta fue positiva y $50 \%$ de los pacientes a los que se les tomó hemograma cursaron con leucocitos mayores a 12.000/ $\mathrm{mm}^{3}$. De los pacientes que presentaron absceso cutáneo, $60 \%$ requirió drenaje quirúrgico.

Setenta y un por ciento de los pacientes recibió un $\beta$-lactámico como tratamiento empírico (56\% como monoterapia y $15 \%$ como terapia combinada con vancomicina). Clindamicina fue prescrita empíricamente en $21 \%$ y vancomicina en $19 \%$ (15\% en combinación con $\beta$-lactámicos y $4 \%$ como monoterapia). Tras obtener el resultado del aislamiento microbiológico, $80 \%$ de los pacientes con SASM completaron el esquema antimicrobiano con $\beta$-lactámicos, $11 \%$ con clindamicina, $7 \%$ con vancomicina y $4 \%$ con cotrimoxazol. Cuarenta y tres por ciento de los pacientes con aislamiento de SARM completó tratamiento con clindamicina y $43 \%$ con cotrimoxazol; $10 \%$ de los pacientes con aislamiento de SARM completó el tratamiento con $\beta$-lactámicos, a pesar de ser cepas resistentes.

Todos los aislamientos fueron sensibles a rifampicina y cotrimoxazol. Ocho por ciento de los aislados fueron resistentes a clindamicina (todos identificados por prueba de difusión en disco positiva); $15,5 \%$ de los aislados fue resistente a eritromicina y $24 \%$ lo fue a tetraciclinas, sin diferencia entre ambos grupos. Por último, 27\% de los aislados de SARM tuvieron una CIM para vancomicina de $1 \mu \mathrm{g} / \mathrm{ml}$.

\section{Discusión}

En nuestro estudio encontramos una prevalencia de $31 \%$ para SARM en IPTB, lo que concuerda con los valores reportados previamente en otras series en nuestra ciudad, que varían desde 10 a 40\%, en pacientes de todas las edades $^{2}$, pero son menores que la descrita en la población pediátrica en otras partes del país ${ }^{7}$ y del mundo, donde SARM representa hasta $60-70 \%$ de las infecciones ${ }^{3}$. Esto es muy importante en el momento de tomar decisiones terapéuticas pues las recomendaciones sugieren emplear antimicrobianos empíricos con cobertura para SARM cuando la prevalencia es mayor a $15 \%{ }^{1,13}$.

Al comparar los aislados de SARM y SASM, sólo alcanzó significancia estadística la diferencia de edad entre los dos grupos, con menor edad promedio en el grupo de SARM. Al evaluar específicamente los lactantes $(<2$ años de edad) encontramos que tenían mayor proporción de infección por SARM, con significancia estadística, lo que también se ha observado en otros estudios ${ }^{7}$. En nuestra experiencia, la edad promedio fue de 6,5 años, mayor que la observada en otras localizaciones geográficas ${ }^{3,11}$, lo que podría explicar el menor número de aislados de SARM en nuestros resultados, comparados con otros.

En estudios previos en niños, no se han encontrado diferencias en el perfil de resistencia de $S$. aureus en pacientes con infecciones cutáneas recurrentes. Nosotros encontramos antecedente de infecciones recurrentes en piel en $14 \%$ de los casos, sin diferencia significativa en el grupo de SAMR.

En Colombia y E.U.A. existen tasas de resistencia a clindamicina cercanas a $20 \% 0^{3,7,11}$, mayor al encontrado en nuestro estudio ( $8 \%$ ); todos los casos de resistencia fueron identificados mediante la prueba de disco D, lo que refuerza la necesidad de realizar esta prueba.

A pesar de que la resistencia de $S$. aureus a tetraciclinas ha disminuido en últimos años, en nuestro medio aún es prevalente; encontramos tasas de $24 \%$ de resistencia. Todos los aislados evaluados conservaron sensibilidad a cotrimoxazol, rifampicina y vancomicina como también se ha reportado en Norteamérica ${ }^{11}$.

La identificación de $10 \%$ de pacientes con aislamiento de SARM, que continuó tratamiento con $\beta$-lactámicos, a pesar de ser resistente, se debe principalmente porque se dieron de alta antes de obtener el antibiograma del aislamiento microbiológico. Adicionalmente podría reflejar la auto-resolución de las IPTB no complicadas ${ }^{1}$.

En los casos de SASM, 11\% completaron el tratamiento antimicrobiano con vancomicina o cotrimoxazol, a pesar de tener opciones de menor espectro de acción, lo que refuerza la necesidad de evaluar el aislado obtenido para lograr el uso racional de los antimicrobianos, con un tratamiento dirigido. En nuestro reporte, en los casos de absceso cutáneo, $60 \%$ de los pacientes requirieron drenaje, porcentaje acorde a lo reportado en otra serie realizada en la Clínica Mayo en E.U.A ${ }^{4}$.

\section{Conclusión}

Ante la alta prevalencia de SARM en IPTB encontrada en esta serie de pacientes pediátricos, se recomienda que el tratamiento empírico de estas infecciones incluya antimicrobianos con adecuada cobertura para el mismo, principalmente para lactantes. Cotrimoxazol y clindamicina parecen ser buenas opciones terapéuticas empíricas.

Agradecimientos: A M. Isabel Múnera. Coordinadora Laboratorio HPTU.

\section{Resumen}

Introducción: El principal microorganismo implicado en las infecciones de piel y tejidos blandos (IPTB) es Staphylococcus aureus, con incremento en las cepas resistentes a meticilina en los últimos años. Objetivo: Identificar la frecuencia de $S$. aureus resistente a me- 
ticilina (SARM) en IPTB en niños que consultaron a un hospital de cuarto nivel en la ciudad de Medellín. Métodos: Estudio descriptivo, retrospectivo, a partir de la revisión de historias clínicas. Se incluyeron pacientes menores de 18 años con IPTB causadas por $S$. aureus que no cumplieran con criterios de enfermedad invasora. Resultados: La prevalencia de SARM en esta población fue de $31 \%$. El principal diagnóstico fue absceso cutáneo (68\%), seguido por infección de sitio quirúrgico (15\%) y celulitis no purulenta (6\%). Tenían alguna co-morbilidad $85 \%$ de los pacientes. Todos los aislados fueron sensibles a rifampicina y cotrimoxazol. Ocho por ciento de los aislados fueron resistentes a clindamicina. Se encontró mayor prevalencia de SARM en lactantes comparado con los mayores de 2 años ( 60 vs 23\%, p = 0,0109). Conclusión: Ante la alta prevalencia de SARM en IPTB se recomienda incluir en el tratamiento empírico antimicrobianos con cobertura para estas cepas, principalmente para lactantes.

\section{Referencias bibliográficas}

1.- Larru B, Gerber J S. Cutaneous bacterial infections caused by Staphylococcus aureus and Streptococcus pyogenes in infants and children. Pediatr Clin North Am 2014; 61 (2): 457-78.

2.- Jiménez J N, Ocampo A M, Vanegas J M, Rodríguez E A, Mediavilla J R, Chen L, et al. CC8 MRSA strains harboring SCCmec type IVc are predominant in Colombian hospitals. PloS One 2012; 7 (6): e38576.

3.- von Specht M H, Gardella N, Ubeda C, Grenon S, Gutkind G, Mollerach M. Communityassociated methicillin-resistant Staphylococcus aureus skin and soft tissue infections in a pediatric hospital in Argentina. J Infect Dev Ctries 2014; 8 (9): 1119-28.

4.- Amin A N, Cerceo E A, Deitelzweig S B, Pile J C, Rosenberg D J, Sherman B M. Hospitalist perspective on the treatment of skin and soft tissue infections. Mayo Clin Proc 2014; 89 (10): 1436-51.

5.- Ray G T, Suaya J A, Baxter R. Microbiology of skin and soft tissue infections in the age of community-acquired methicillin-resistant Staphylococcus aureus. Diagn Microbiol Infect Dis 2013; 76(1): 24-30.

6.- Lozano J S, Sebastián M S, González F, Hernández-Sampelayo T, Gómez M L N. Infecciones bacterianas de la piel y tejidos blandos. Protocolos de infectología AEP. [Internet]. 2011 [Citado en abril de 2016]. Disponible en: http://www.aeped.es/sites/ default/files/documentos/piel.pdf

7.- Sosa Ávila L M, Machuca Pérez M A, Sosa Ávila C A, González Rugeles C I. Infecciones por Staphylococcus aureus meticilino resistente en niños en Bucaramanga Colombia. Salud UIS 2010; 42 (3): 248-55.

8.- Bassetti M, Baguneid M, Bouza E, Dryden M, Nathwani D, Wilcox M. European perspective and update on the management of complicated skin and soft tissue infections due to methicillin-resistant Staphylococcus aureus after more than 10 years of experience with linezolid. Clin Microbiol Infect 2014; 20: 3-18.

9.- Dryden M S. Skin and soft tissue infection: microbiology and epidemiology. Int $\mathrm{J}$
Antimicrob Agents 2009; Suppl 1: S2-7.

10.- Kaplan S L. Implications of methicillinresistant Staphylococcus aureus as a community-acquired pathogen in pediatric patients. Infect Dis Clin North Am 2005;19 (3): 747-57.

11.- Karamatsu M L, Thorp A W, Brown L. Changes in community-associated methicillin-resistant Staphylococcus aureus skin and soft tissue infections presenting to the pediatric emergency department: comparing 2003 to 2008. Pediatr Emerg Care 2012; 28 (2): 131-5.

12.- $\mathrm{CDC} / \mathrm{NHSN}$ Surveillance Definitions for Specific Types of Infections; 2014. Centers for Disease Control and Prevention. [Internet]. Enero 2017. [Citado en febrero de 2017]. Disponible en: https://www.cdc.gov/nhsn/pdfs/ pscmanual/17pscnosinfdef_current.pdf.

13.- Comité Nacional de Infectología, Moyano M, Peuchot A, Giachetti AC, Moreno R, Cancellara A, et al. Skin and soft tissue infections in children: consensus on diagnosis and treatment. Arch Argent Pediatría 2014; 112 (2): 183-91. 